\title{
Kant in neuer ultramontan- und liberal-katholischer Beleuchtung.
}

Kritisch gewürdigt von Bruno Bauch.

In Bd. XXII, Heft 1 des Jahrbuchs für Philosophie und spekulative Theologie ist ein Aufsatz erschienen unter dem Titel: "Kant, der Philosoph des Protestantismus". Als Herausgeber des Jahrbuchs zeichnet Dr. Ernst Commer, päpstlicher Hausprälat. Verfasser des genannten Aufsatzes ist ebenfalls ein Prälat, nämlich der Herr Prälat, Kanonikus Dr. Michael Glossner. Ein Aufsatz über den Philosophen des Protestantismus in der Zeitschrift eines päpstlichen Hausprälaten, aus der Feder eines anderen Prälaten hat inmerhin etwas Überraschendes. Herr Commer hat sich in der jüngsten Zeit einen Namen gemacht; nicht wie man nach dem Titel seiner Zeitschrift erwarten dürfte, auf dem Gebiete der Theologie oder etwa dem der Philosophie, überhaupt nicht auf dem der Wissenschaft. Der päpstliche Hausprälat verdankt seinen jungen Ruhm im Gegenteil einer heftigen Anfeindung des Versuches freierer Regung $\mathrm{zu}$ wissenschaftlichem Denken innerhalb der Theologie seiner Kirche. $\mathrm{Er}$ richtete sich gegen den verstorbenen katholischen Theologen Hermann Schell, der zwar freier denken mochte, als dem Prälaten Commer lieb war, dessen Versuche, dem Katholizismus wenigstens ein Geringes von kulturellem Leben zuzuführen, jeder objektiv über der Sache Stehende aber nicht gerade als allzu kühn und wagemutig wird bezeichnen dürfen. Und doch bekämpfte ihn Commer in einer Weise, die wir hier zwar nicht näher zu charkterisieren brauchen, die ihm aber seinen nicht gerade beneidenswerten Ruhm eingebracht hat. In eben dieses Herrn Prälaten Commer Zeitschrift also erschien ein Aufsatz von dem anderen Prälaten Glossner über das Thema „Kant, der Philosoph des Protestantismus".

Nun wir sagen nicht: es wird sich jeder im voraus denken können, was Herrn Glossners Aufsatz für ein Machwerk sei. Wir 
sagen es nicht, obwohl wir wissen, dass derselbe Prälat Commer sich von demselben Prälaten Glossner in einem zweiten Artikel desselben Heftes der Zeitschrift, das den Kant-Artikel enthält, ein hohes Lob auf seine Heldentat gegen den toten Schell hat singen und gefallen lassen. Trotzdem sage ich nicht, es könne sich jeder im voraus denken, was es mit Herrn Glossners Kant-Artikel auf sich habe. Das kann sich in der Tat niemand im voraus ausdenken, nicht etwa, weil dieser Artikel den Erwartungen, die man an die bereits bekannten, soeben berichteten Tatsachendaten zu knüpfen geneigt sein möchte, widerspräche, sondern vielmehr deshalb, weil er sie alle, und wären sie noch so hoch gespannt, übertrifft.

Der Herr Verfasser ist auch Doktor. Hätte er das seinem Artikel nicht ausdrücklich beigefügt, so würde ich vermutet haben, dieser KantAufsatz sei seiv erster schüchterner Versuch zu "wissenschaftlicher" Betätigung; schüchtern natürlich nur der Sache nach. Da ist denn aber auch dieser Versuch zu wissenschaftlicher Betätigung in der Tat und in der Wahrbeit gleich der sachlich schüchternste, d. h. unwissenschaftlichste und unglücklichste, der mir jemals vorgekommen ist. Der Begriffswirrwarr ist - ich rede immer nur von der Sache - so grob, roh und verschroben, dass jeder mittelmässig begabte, in der Tertia eines deutschen Gymnasiums sitzende Knabe, wollte er den Ansprüchen eines einigermassen tüchtigen Lehrers genügen, den Stoff seines Aufsatzes in ganz anderer Weise sichten und anordnen müsste als der Herr Kanonikus Prälat Dr. Michael Glossner.

Und woher nimmt seine Abhandlung den Stoff über Kant, den Philosophen des Protestantismus? Man sollte erwarten: aus Kants Werken selbst. Aber auch hier hätten wir schon unsere Erwartungen zu hoch gespannt. Paulsens und Kaftaus gleichnamige Aufsätze und Falckenbergs kleines Hilfsbuch der Geschichte der neueren Philosophie seit Kant, sowie Euckens kurzgefasste Beiträge zur Geschichte der neueren Philosophie, nicht aber die grösseren Werke dieser Autoren hat diese Arbeit benutzt. Und wie hat sie sie benutzt! Aus ihnen allen werden nach Gutdünken die gerade beliebten Materialien herausgerissen. Diese werden verkümmert, verzerrt, verschoben und endlich in eine eigene oder besser: so eigenartige Form gebracht, dass sie jedem an etwas Besseres Gewöhnten einen nicht gerade gelinden Ekel erregen 
muss. Das ist dann das Kind des Geistes. Getauft wird es auf den Namen: „Kant, der Philosoph des Protestantismus“. IVir fragen noch nicht: Ist dieser "Kant" des Herrn Glossner auch der Kant, wie ihn die Forschung und Wissenschaft kennt? Fragt man auch nur ganz bescheiden, inwieweit in diesem Machwerk überbaupt von Kant die Rede ist, so müssen wir gleich antworten: erstaunlich wenig, so wenig, dass für das gedankenlose Compilat auch bätte jeder andere Name berbalten können. Ist doch in dieses hineingezerrt, was aus der ganz oberflächlichen Lektüre der erwähnten Autoren gerade in die inkonsistentesten Vorstellungsmassen eingegangen war.

Wie nun ist es mit dem Wenigen bewandt, das wir über Kant selbst hier zu hören bekommen?

Seinen Gewährsquellen tritt der Artikel, da er sie ja in erster Linie bekämpft, nachdem er ihre Wissenschaft zu seiner Weisheit entstellt, mit einem vollkommen selbständigen, souveränen - wir wollen keinen stärkeren Ausdruck gebrauchen Unvermögen des Verständnisses gegenüber. So beginnt er gleich mit einer Art von Polemik gegen Kaftans Behauptung, dass „Kant seine Abkunft von Luther nicht werde verleugnen wollen ${ }^{\text {. }}$. Denn er knüpft daran die kritisch seia sollende Bemerkung, dass jene Behauptung "etwas zaghaft" klinge. Sei doch „der Glaube im Sinue Luthers etwas ganz anderes als der moralische Vernunftglaube Kants“. Auf Grund des Kantischen Rationalismus, d. h. deshalb, weil Kant „von einer Offenbarung, einer anderen Walurheitsquelle, als der Vernunft nichts wissen" wolle, sucht Herr Glossner zunächst also den Gegensatz von Luther und Kant gegenüber Kaftan - zu verschärfen. Man mag dabei über Kaftaus Arbeit denken, wie man will, nach den kurzen Geistesproben kann über Herrn Glossners Arbeit jetzt schon, in zwei Punkten wenigstens, kein $Z$ weifel mehr bestehen: ersteus trägt sie an Luther wie an Kant den Begriff des Rationalismus sowohl, als denjenigen der Offenbarung heran, wie er in streng ultramontaner Reinkultur grossgezogen ist; zweitens begegnet sie dem positiven Verhältnis von Luther und Kant mit einer ebenfalls in ultramontaner Reinkultur grossgezogenen Verständnislosigkeit. Zweifelhaft bleibt aber zunächst nur, ob diese Verständnislosigkeit grösser ist gegenüber der Tat Luthers oder gegenüber derjenigen Kants. Aber auch das entscheidet sich sehr bald. 
Was zunächst Kant anlangt, so wird erklärt, die Vernunft im Kantischen Sinne, oder wie unser Autor zu sagen beliebt, die "Kantische Vernunft", sei "subjektivistisch", so subjektivistisch, dass Kant die "Objektivität unseres intellektuellen Erkennens", wie wiederum unser Autor sagt, „entschieden verwirft". Herr Glossner kennt also nicht einmal das fundamentalste Problem der Kantischen Vernunftkritik, das gerade die Gegenständlichkeit, die Objektivität des Erkennens zum Gegenstande selber hat. Er hat keine Ahnung davon, dass der Sinn der Vernunftkritik doch gerade auf die Aufdeckung objektiver Erkenntniskriterien geríchtet ist. Das heisst: er hat nicht die leiseste Ahnung von der elementarsten und fundamentalsten Prinzipienfrage Kants. So erweist es sich denn schon an der Kantischen Fragestellung, dass Glossners Verstäudnislosigkeit Kant gegenüber die radikalste ist, die sich nur ausdenken lässt. Was sodann die Tat Luthers anlangt, so zeigt sich genau ebenso, dass ihm deren Sinn und Bedeutung vicht minder fremd geblieben ist, als der Sinn und die fundamentale Bedeutung der Kantischen Philosophie. Was er über Luther sagt, wirkt geradezu grotesk. Wir hörten, dass nach Herrn Glossner der Rationalismus das Trennende zwischen Luther und Kant sein solle. Weil er aber von Kant gering denkt und nicht weniger gering vom Protestantismus, möchte er zunächst selber Kant doch gern als Philosophen des Protestantismus gelten lassen. Er kann das nicht besser erreichen, als durch eine Herabsetzung Luthers. Dazu muss er aber Luthers Rückkehr zum Evangelium - ich scherze nicht - einfach leugnen. Um das wieder tun zu können, schlägt er seinem Argumente von der ersten Seite schon auf der zweiten vollkommen ins Gesicht. Gegen Paulsen sagt unser Argumentator: „Schon der Umstand, dass Luther im Rationalismus und Naturalismus der Renaissance Bundesgenossen fand, hätte ihn zur Vorsicht mahnen sollen." Hier soll also offenbar nun Luthern doch mit dem Rationalismus und Naturalismus Verwandtschaft vindiziert werden. Ja, damit ist es nicht genug. Luthers Rechtfertigungslehre wird geradezu zum Naturalismus. Wer es nicht für möglich hält, dass jemand einen solchen verschrobenen Gedanken fassen könne, der lese den einen köstlichen Satz: „Der anscheinend exzessive Supernaturalismus der Rechtfertigungslehre Luthers ist in Wahrheit Naturalismus." Und wenn man die daran unmittelbar anknüpfende Folgerung: „Mit der Rückkehr zum alten Evangelium ist es demnach nichts, “ d. h. 
eben die direkte Leugnung von Lutbers Rückkehr zum Evangelium liest, so ergiebt sich mit zwingender logischer Notwendigkeit: Herr Glossner ist nicht etwa bloss ausser Stande, einen Gedanken von einer Seite bis zur anderen festzuhalten, ohne ihn durch kontradiktorischen Gegensatz aufzuheben, er hat bei diesem Unvermögen zu den einfachsten Regeln der formalen Logik auch nicht die leiseste Abnung vom inhaltlichen Sinn und der Bedeutung von Luthers Tat. In ihrem einfachsten Kern und Wesen ist ihn diese so sehr verschlossen, dass sich schon jetzt, was zuerst noch problematisch war, ob das Unvermögen des Verständnisses - ich brauche auch jetzt den mildesten Ausdruck, der sich für die Charakteristik dieser Arbeit finden lässt - gegenüber der Tat Luthers oder derjenigen Kants grösser ist, entscheidet: Dieses Unvermögen $\mathbf{z u}$ verstehen, ist in beiden Fällen gleich radikal, weil es hier wie da überhaupt das radikalste ist, das sich nur ausdenken lässt.

Es würde empörend wirken, wäre es nicht gar zu lächerlich, wie hier der furor antiprostentanticus et antikantianus sich geberdet. Erst trennt nach seiner Regel der Rationalismus Luther und Kant, dann muss Luther sogar schon „im Rationalismus und Naturalismus der Renaissance Bundesgenossen finden", um schliesslich selber Naturalist zu werden, bis, da Luthers Glaube nun doch wieder "etwas ganz anderes ist als der moralische Vernunftglaube Kants“, endlich inbezug anf Luther und Kant erklärt wird: "die Ähnlichkeit reduziert sich auf den gemeinsamen Subjektivismus*. Diesmal meint Herr Glossner nun den moralischen „Subjektivismus", um sogleich eine wahrhaft tragikomische Begriffsposse aufzuführen. Er erlässt auf der einen Seite an den Protestantismus die possierliche Warnung vor Kant: „Der Gewinn aber, den der Protestantismus aus der philosophischen Begründung durch Kant zu schöpfen vermag, ist ein höchst zweifelhafter und bedenklicher; denn die von Kaftan betonte sittliche Erfahrung spieit bei Kant eine Rolle, die geeignet ist, die Gottheit selbst vom Throne zu stürzen und an ihre Stelle die sittliche Weltordnung zu setzen, was bekanntlich von Fichte geschehen ist.“ Auf diese klägliche, im miserabelsten Ultramontanendeutsch vorgetragene Expektoration folgt aber andererseits gleich die Erklärung, dass Kant und der Protestantismus eigentlich doch einander wert seien und zusammengehören, indem ebenso wie das Prinzip der Kantischen Pbilosophie, auch dasjenige des Protestan- 
Kant in neuer ultramontan- $u$. liberal-katholischer Beleuchtung.

tismus „einer schrankenlosen Willkür Tür und Tor offne, sodass die Gleichheit der Schicksale des Protestantismus und des Kantianismus ihre Verbindung auch von dieser Seite rechtfertigt, eine Verbindung, die aber keineswegs dem einen wie dem anderen zum Vorteil und zur Empfebling gereicht."

Der Herr Kanonikus weiss nicht, oder will es nicht wissen, dass das von Luther dem Protestantismus gewiesene Prinzip die Willkür des „Pfaffentums“, um einen Ausdruck Kants zu gebrauchen, gebrochen und in der sittlichen Freiheit der Welt das objektiv-sittliche Prinzip gebracht, er weiss nicht, dass Kant ebendiesem objektiv-sittlichen Prinzip in der Lehre von der Autonomie die objektive philosophische Begründung gegeben. Wenn er also davon spricht, dass dieses Prinzip „einer schrankenlosen Willkür Tür und Tor öffne", so bringt er es selbst noch einmal ungewollt und unabsichtlich auf eine klare und bündige Formel, dass das ultramontane Unvermögen, zu verstehen, gleich radikal ist gegenüber der reformatorischen Tat Luthers, wie der philosophischen Tat Kants. Trotzdem aber wagt man es, vom Standpunkte des Ultramontanismus aus, über Kant, den Philosophen des Protestantismus, zu schreiben.

So also stellen sich Luther und Kant in dem Urteile der ultramontanen "Wissenschaft" dar. Viel ist sonst von Kant nicht mehr die Rede, aber auch in dem Wenigen, das noch von Kant handelt, steckt eine solche Überfülle des Absurden und Sinnwidrigen, dass ich gestehen muss: Es ist nur eine Art der ultramontanen Befähigung, die meine nneingeschränkte Bewunderung erregt hat, die Fähigkeit nämlich, in so wenig Worten so viel des horrendesten Unsinns zu reden. Da kommt Herr Glossner z. B. auf Kants Widerlegung der Gottesbeweise - ganz abrupt und zusammenhangslos - zu reden, und er behauptet, dass „Kants Kritik auf erkenntnistheoretischen Ansichten beruht, deren Falschheit längst nachgewiesen ist. ${ }^{*}$ - ${ }_{n}$ Falschheit ${ }^{4}$ ? -. „Längst nachgewiesen?" - Was? - Wielange? - Wann? - Von wem? Vielleicht von Herrn Glossner? Warum hat er uns bier den Nachweis vorenthalten and uns einige Minuten herzlichsten Humors missgönnt? Indes für seine Zwecke hat er diesen Nachweis wohl nicht nötig. Da sein Machwerk nun einmal die Druckerei passiert, in einer öffentlichen Zeitschrift Aufnahme gefunden hat, wird es anch Leser finden, denen die Art, ohne Beweise zu argumentieren, imponiert, obwobl sie uns theoretisch ein hölzernes Eisen and 
praktisch noch schlimmer als das erscheint. Oder, wie bringt man es fertig, von Kant, nachdem er doch zum Subjektivisten gestempelt ist, zu erklären, dass er sogar „das Allgemeine zu hypostasieren" suche? Das wissenschaftliche Unvernögen hat seine eigenen Künste, scheints.

Damit genug vom Kant des Herrn Glossner. Man sieht, in diesem Bilde ist kein Zug vom Kant der Geschichte, wie ihn die Wissenschaft kennt, und im Kant der Geschichte darum auch kein Zug von dem Bilde, wie es die ultramontane Phantasie des Herrn Glossner sich malt.

Diese bat auch um die ganze nachkantische Philosophie ihre witzlosen Sagen gesponnen. Wenn wir auch nicht allen Seitensprüngen dieser Art "wissenschaftlicher" Possenreisserei, die dabei aufgeführt werden, unsere Aufmerksamkeit schenken dürfen, um nicht vom eigentlich Kantischen Thema abzukommen, so müssen wir doch wenigstens auf das noch mit einem Wort eingehen, was unser Autor selbst als direkte Wirkung der Kantischen Philosophie ansieht.

Zunächst meint er, dass sich in der Mannigfaltigkeit der von Kant ausgegangenen Richtungen die "Zerfahrenheit der Anschau* ungen in der protestantischen Welt" widerspiegele. Die geistigsittliche Vornehmheit, die in diesem geschmackvollen Ausdruck liegt, brauchen wir nicht zu kritisieren. Es ist genug, diesen Ausdruck, der den Autor selbst kritisiert, zu verzeicbnen. Zur Sache aber mag auch die Bemerkung genügen: Mit diesem römischultramontanen Blicke kann vur der absolut Arme im Gejste den Reichtum des deutschen Geisteslebens anschielen. Denn dieser Geistesreichtum, das wird Herr Glossner freilich ebensowenig begreifen, wie die deutsche Geisteskultur selbst, beruht in letzter Linie anf dem protestantischen Prinzip, das jener so zu schmähen beliebt. Dieser Protestantismus ist freilich etwas Anderes, als das, was Herr Glossner so nennt. Denn er hat in letzter Linie auch keine Ahnung davon, was Protestantismus ist. Er kommt von dem beschränkten Gedanken des Konfessionalismus nicht los. Er versteigt sich zu der Warnung: „Indem man Kant als den Philosophen des Protestantismus feiert, giebt man den Anspruch anf Universalität, auf allgemeine Gültigkeit, die vom Begriffe der Wissenschaft unzertrennlich ist, und somit den Anspruch anf Wissenschaftlichkeit preis." Fr meint also, Kant als Philosophen 
des Protestantismus anzusehen, bedeute eine Konfessionalisierung der Wissenschaft. Und davor will er warnen, eine Warnung, die sich gerade aus seinem Munde allerliebst ausnimmt. Er will jetzt anch nichts mehr davon wissen, dass man Kant als Philosophen des Protestantismus ansehe, er, der soeben selbst dafür plädierte. Aber was will er eigentlich? Zuerst sucht er durch verschrobene Begriffe von Rationalismus und Offenbarung einen Gegensatz von Luther und Kant aufzurichten, der nicht vorhanden ist. Dann will er trotzdem Kant als Philosophen des Protestantismus gelten lassen. Bald aber warnt er den Protestantismus vor der Kantischen Philosophie, um sofort zu erklären, „dass die Gleichheit der Schicksale des Protestantismus und des Kantianismus ihre Verbindung auch von dieser Seite rechtfertigt". Bald wieder warnt er umgekehrt die Kantische Philosophie vor dem Protestantismus, damit jene nicht dem Konfessionalismus verfalle und die Universalität einbüsse. Was soll das, was wollen diese ewigen plumpen, einfältigen Widersprüche? Der Herr Prälat weiss wenig, wissenschaftlich weiss er nichts, rein nichts von dem, worüber er schreibt. In dem Falle aber weiss er wenigstens, was er will. Er will den Protestantismus und den Kantianismus soviel er kann, herabsetzen, bietet alle für diesen seinen heiligen $Z$ weck erreichbaren Mittel auf, tut alles, was ihm in diesen seinen Kram passt. Aber da er nun einmal von alledem wissenschaftlich nichts, rein nichts weiss, so weiss er auch nicht, dass das, was ihm in seinen Kram passt, gar nicht zu den Gesetzen der Logik passt. Er hat nicht einnal ein Gefübl dafür, dass er beständig gegen das einfache Widerspruchsgesetz verstösst, die logische Blindheit und Unfähigkeit in wissenschaftlichen Dingen lässt ihn bei jedem zweiten Schritt in einen dritten Widerspruch fallen. Seinen redlichen Willen, Protestantismus und Kantianismus zu schmähen und zu verlästern, könnte nur ein selbst unredlicher Wille bezweifeln. Denn jene seine redliche Tendenz ist zu offenbar geworden. Aber ebenso offenbar ist es, dass sein unzweifelhafter Wille nicht im Stande ist, mit Gründen gegen Protestantismus und Kantianismus etwas auszurichten. Mit Gründen, - das will er ja freilich nicht, er kann es auch nicht, das können ja auch andere Leute als er nicht, der er überhaupt in diesen Dingen nichts wissenschaftlich kann. Er weiss ja, wie wir sahen, nicht einmal, was Protestantismus ist. Lasse er sich das wenigstens vom Philosophen des Protestantismus sagen, dass zwischen dem protestantischen 
Prinzip und dem. Konfessionalismus, die er nämlich mit einander verwechselt, um auf dieser Verwechselung das Nest von Widersprüchen aufzubauen, ein himmelweiter Unterschied ist. Diesen Unterschied wird er freilich nie und nimmer begreifen. Aber hätte er Kants "Religion innerhalb der blossen Vernunft", die er zwar schmäht, aber doch nicht kennt, wenigstens gelesen, so möchte er zum mindesten vielleicht soviel wissen, dass man nach Kant Protestant sein könne, ohne etwa zu einer sichtbaren kirchlichen Anstalt des Protestantismus zu gehören. Dass das auch ein echt Lutherischer, in Luthers Idee der unsichtbaren Kirche zum Ausdruck gelangender Gedanke ist, das weiss er natürlich auch nicht. Und wenn er es schon wüsste - die Aufnahme auch dieses Gedankens läge wohl schliesslich jenseits der Grenzen seines Könnens. Wenn er von einer "Zerfahrenheit der Anschauungen in der protestantischen Welt" redet, so zeigt er nur, wie ferne ihm auch nur die Vorstellung von einer innerlichsten Überzeugung, nicht nur die innerlichste Überzeugung selbst, liegt. Die Wahrheit, die für die Wissenschaft eine ewige Idee und das heisst in Kants Sprache eine, ewig lebendige Tätigkeit fordernde, Aufgabe ist, wird für den, dem der Gedanke der Wissens- und Gewissens-Freiheit nicht aufgegangen ist, zu einer toten Sache, die eine Art von tyrannischer Gedankenpolizei mag zu hüten glauben, weil sie ebenfalls glauben mag, die Wahrheit lasse sich einfach beschliessen, ohne dass man sie erarbeitet, die aber nimmer Sache überzeugungsvoller Gesinnung und geistiger Kultur sein kann. Darum muss natiirlich alles, was Herr Glossner über die grossen nachkantischen Systeme, die den allgemeinen Kulturbegriff gerade unendlich $\mathbf{z u}$ bereichern und zu vertiefen berufen waren, zu sagen wagt, nicht nur abermals den Stempel radikalsten Unvermögens $\mathrm{zu}$ verstehen tragen, sondern geradezu ins Kindische ausschlagen. Am deutlichsten wird das bei des Autors Meinungsäusserung über Hegel und namentlich über Fichte, der nach Herrn Glossners tiefsinnigem Urteil es unternommen haben soll, „den Menschengeist dem ,Absoluten“ gleichzusetzen“. Fichte wird bei Herrn Glossner zum Pantheisten, ja zum Atheisten. Daran ist ihm natürlich in letzter Linie Kant ebenso schuld, wie an Jacobis "Theosophie“ und "Mystizismus“. Ja die ganze böse philosophische Entwickelung, die in Nietzsche nach unseres Autors bewährter „wissenschaftlicher" Sinnigkeit und Findigkeit „richtig bei der Bestie angelangt" sei, ist in letzter Linie Kants Ver- 
Kant in neuer ultramontan- $u$. liberal-katholischer Beleuchtung.

schulden. Mit dieser letzten Wendung erhält natürlich auch der „Darwinismus" die quittierende Glosse Glossners.

Nun gewiss geht die ganze philosophische Arbeit seit Kant, sofern sie selbst einen philosophischen Wert in der. Geschichte beanspruchen darf, auf Kant zurück. Nur stellt sich, eben weil sie einen Wert hat, diese Entwickelung in Wahrheit etwas anders dar, als im Urteile des Herrn Glossner. Wer für Luther, der eine seit den Tagen Jesu von Nazareth nie wieder erlebte sittlich religiöse Bereicherung der Menschheit gebracht, wer für Kant, der eine seit Platon nie wieder erlebte gedankliche Vertiefung erarbeitet, nur verletzende Entwürdigung und Entstellung aufzubringen weiss, wer die Männer, die den ewigen Stolz der ganzen an der fortschreitenden Kultur beteiligten Menschheit bilden, nur mit Kot und Schmutz zu bewerfen strebt, indem er ihren Glauben, ihre reformatorische Tat herabzusetzen trachtet, wessen persönliche Neigung darum so gar nichts vom Fortschritt, sondern alles immer nur vom Stillstand wissen will, dem muss natürlich alle Entwickelung ein Graus und Grenel sein; die geistesgeschichtliche noch mehr als die organologisch-physische. Aber auch diese braucht wirklich den Nenschen nicht, wie Herr Glossner erklärt „bei der Bestie angelangt" sein zu lassen. Diese Furcht hörte ich einmal in einer entwickelungstheoretischen Diskussion - es war hier in Halle - von einem Zoologen durch ungefähr folgende treffende Bemerkung beschwichtigen: die Annahme der Entwickelung braucht nicht umgekehrt zu bedeuten und kann nicht bedeuten, dass der Mensch sich wieder zum Affen, hinabentwickeln' sollte. - Nein, das ist wahrlich nicht nötig, ob es manchmal auch so scheinen mag. ${ }^{1}$ )

Vielleicht ist dieser wissenschaftliche Gedanke ein Trost für die Wissenschaftsfeindschaft Herrn Glossners. Ich würde mich freuen, mit diesem Troste von seinem Machwerk, von dem ich nun eigentlich Abschied nehmen muss, zu scheiden. Sein Elaborat hat ausser der theoretischen Seite freilich auch noch eine praktische. Es ist eine antiprotestantische und antiwissenschaftliche Tendenzschrift übelster Sorte. In das moralische Gebiet fällt es im Einzelnen z. B. schon, wenn man Kant und dem Protestantis-

1) Tăusche ich mich nicht, so hat übrigens auch schon Lotze, an dem unser Autor ja noch etwas Gutes zu finden meint, denselben Gedanken ganz s.hnlich ansgedritckt. 
mus vorwirft, dass ihr „Prinzip einer schrankenlosen Willkür Tür und Tor öfne", wenn sich Herr Glossner erdreistet, vom Reformator Luther zu reden und labei das Wort "Reformator" in Anführungsstriche $\mathrm{zu}$ setzen, ein direkt infames Verfahren, das seinen Gipfel da erreicht, wo es auch auf den Glauben Luthers angewandt wird. Wer das lesen kann, ohne dass ihm im Namen der Menschheit die tiefste Zornes- und Schamröte aufsteigt, der kann an der Idee der Menschheit keinen Teil haben. Möchte darum theoretisch des Herrn Kanonikus furor antiprotestanticus, wie ich vorhin sagte, empörend wirken, wenn er nicht so lächerlich wirkte, so muss er dagegen unter praktischem Gesichtspunkte empörend wirken, trotzdem er auch gar so lächerlich wirkt. Nach den oben gegebenen Tatsachenproben wird man freilich erst nicht mehr fragen dürfen: wo bleibt hier die in dem obersten christlichen Sittengrundsatz eingeschlossene Achtung vor dem Nächsten und seiner Überzeugung? Nach jenen Proben wäre diese Forderung freilich schon zu hoch gestellt. Wer Luthers Glauben als unechten Schein verdächtigt und Kants Vernunftreligion herabsetzt, der kann an jener Forderung nicht mehr gemessen werden. Aber stellen wir jetzt unsere Forderung auch noch so tief, und fragen wir bloss: wo bleibt hier die vom Ultramontanismus so gepriesene "Toleranz"? - so weiss man gleich, wie es um diese "Toleranz" bestellt ist. Die dem Anderen zugemutete Toleranz der eigenen Intoleranz hat nicht nur nicht in der Politik, sondern auch nicht in der Philosophie, weder in der theoretischen, noch in der praktischen, einen logischen Ort. Man braucht nur das blosse Faktum des Aufsatzes reden zu lassen, so weiss man, dass man sich jede moralische Beurteilung, ob der deutlichen Sprache dieses Aufsatzes, ersparen kann. Darum habe ich mich von vornherein auch nur mit dem Werk, nicht aber mit dem Werkmeister, um eine Unterscheidung Luthers anzuwenden, beschäftigt. Denn das Werk spricht sich selbst das Urteil: Dass es überbaupt existiert, dass es nicht bloss geschrieben, sondern auch gedruckt, und das nicht bloss auf die alleinige Verantwortung seines Urhebers, dass es vielmehr auch in einer Zeitschrift Aufnahme finden und einem sicheren Leserkreise zugänglich gemacht werden konnte - das alles ist und bleibt beschämend und empörend. Darum wollen wir es uns auch tatsächlich ersparen, die moralische Seite der Sache zu beurteilen. Im Urteile jedes gerecht und billig denkenden Lesers wird diese sicb in ihrem Wesen rein und unverfälscht darstellen schon durch 
die theoretische Kenntnis dieses echten Dokumentes ultramontaner "Wissenschaft".

Ich erwähnte vorhin, dass in demselben Hefte der Zeitschrift des Herrn Commer ebenfalls aus der Feder des Herrn Glossner, ausser dem antikantischen Machwerk, ein den Commerschen SchellHandel glorifizierender Artikel enthalten sei. Nun fürchte man nicht, dass ich Herrn Glossners Pfaden weiter folgen möchte. Sie interessieren mich im weiteren herzlich wenig, habe ich doch auch schon von ihnen Abschied genommen. Ich bin ja dem Wege, den sein Geist genommen, auch bisher nur gefolgt, weil er sich auf ein Gebiet verirrt hatte, auf dem er nichts zu suchen hat, und nicht Herrn Glossners, sondern dieses Gebietes wegen tat ich das. Einen Geist, wie den Herrn Glossners, musste ich von diesem Gebiete verweisen, schon der wissenschaftlichen Sauberkeit wegen. Das war alles. Sein zweiter Artikel kümmert mich schon rein gar nichts mehr. Er hatte höchstens ein mittelbares Interesse. Ausser Schell und Kraus sucht Herr Glossner nämlich gegen einen Katholiken seine bannenden Donnerschläge zu richten, der vor einiger Zeit auch einmal $\mathrm{zu}$ Kant Stellung genommeu hatte.

Eine würdigere Stellung zu Kant freilich nimmt die Arbeit dieses anderen katholischen Autors ein. - Ich meine die Schrift "Katholischer Glaube und die Entwickelung des Geisteslebens" von Dr. Karl Gebert. ${ }^{1}$ ) „Dieser Vortrag will den Kampf des religiösen Katholizismus gegen den politischen philosophisch-wissenschaftlich rechtfertigen." Mit diesen Worten kennzeichnet der Autor von vornherein sein Unternehmen. Die klare und unzweideutige Unterscheidung zwischen religiösem und politischem Katholizismus hat mit besonderem Nachdruck in unserer Zeit Franz Xaver Kraus zur Geltung gebracht. In der Kraus-Gesellschaft zu München ward die Arbeit zunächst mündlich vorgetragen. Man weiss also auch von ihr im voraus ungefähr, was man zu erwarten hat. An der katholischen Rechtgläubigkeit des Verfassers kann höchstens der Ultramontanismus zweifeln. Denu der Verfasser nimmt der Religion gegenüber die einzig mögliche Stellung, nämlich die vollkommenster "Gewissensfreiheit" ein. "Der Glaube ist

1) Katholischer Glaube und die Entwickelung des Geisteslebens. Öffentlicher Vortrag, gehalten in der Krausgesellschaft in München am 10. Januar 1905 von Dr. Karl Gebert. München 1905 Selbstverlag der Krausgesellschaft. Kommissionsverlag: St. Bernhards Verlag. 
ihm ein rein persönliches Verbältnis zu seinem Gott ${ }^{4}$, er vertritt also einen persönlichen, innerlichen Überzeugungsglauben, den der Ultramontane, der nicht weiss, was Überzeugung ist, in Anführungsstriche setzen muss, sobald er von Luther redet. Dem religiösen Katholizismus gegenüber wird sich der politische kaum anders verhalten, was aber nicht bedeuten soll, dass wir jenen mit Luthern etwa identifizieren. Bekennt doch Gebert ausurücklich seine "Liebe zum angestammten Glauben und die feste $\mathrm{Zu}$ versicht auf die religiöse Lebenskraft des Katholizismus". Wie man über diese auch immer sonst denken möge, die vom Autor geforderte Gewissensfreiheit soll ihm ungeschmälert bleiben. Sein persönlicher Glaube, für den er sie fordert, soll hier unverletzlich sein. Und wenn ich auch Gebert gegenüber die Kritik zum Schluss nicht unterdrücken werde, so mag er im voraus überzeugt sein, dass sie nie seine persönliche Religiosität, die ich für echt und tief halte, betreffen werde, sondern allein seine Religionsphilosophie, die als Philosophie der Kritik nicht entraten kann, wie ich vorhin mich ja auch nur gegen die freilich wunderliche Philosophie des Ultramontanismus wandte. Dabei habe ich immer nur Geberts Beziehung zu Kant im Sinne und kann manchen seiner Untersuchungen nicht im Einzelnen folgen, da sie unseren Interessen, wenigstens denen dieser Zeitschrift zu fern liegen, so interessant und charakteristisch sie auch an und für sich sein mögen. So ist ja die Hauptabsicht der Schrift, die Auseinandersetzung mit dem Ultramontanismus, d. h. dem politischen oder wie der Autor noch sagt, dem "Zentrums-Katholizismus" von grösstem allgemeinem Interesse. Man muss diese verderbliche und gefährliche Tendenz so genau kennen, wie der Autor, um sie mit seiner Sachlichkeit in ihren kultur- und religionsfeindlichen Machenschaften darstellen zu köunen. Von ihren Vertretern sagt Gebert treffend: sie „erklären der Kultur die Feindschaft, ${ }^{1}$ ) den Krieg kann man nicht sagen, weil der Krieg ein Geisteskampf sein müsste, der ein Eingehen auf das Wesen der Kultur voraussetzen und ein Kampf mit gleichen Waffen sein müsste". Und mit unwiderleglichem Recht sagt er von dieser Tendenz, der ${ }_{n}$ der Zentrums-Wahlzettel als Zeichen echten Christentums gilt“, dass sie „das Gegenteil wahrer Religiosität" sei ; oder „der Ultramontanismus ist ja nicht bloss

1) Vom Autor selbst gesperrt. 
kulturwidrig, ${ }^{1}$ ) sondern auch unchristlich". ${ }^{1}$ ) Dass Gebert dem Archaismus der thomistischen Lehre ebenso ablehnend gegenübersteht, wie er sich als Glied des ganzen modernen Kulturzusammenhanges fühlt und mit ganzer Seele als deutscher Mann innerhalb des deutschen Geisteslebens arbeiten und wirken will, das versteht sich danach wohl von selbst. Auf alle scine Darlegungen im Einzelnen einzugehen, würde uns, wie gesagt, zu weit führen. Was uns aber näher beschäftigen soll, das ist seine Stellung zu Kant, die in allen Punkten glücklicherweise gerade das Gegenstück zu derjenigen des Ultramontanismus ist.

Gilt dem ultramontanen Katholizismus Kant als Gefahr und Verderbnis, so weiss der Vertreter des liberalen Katholizismus Kants Philosophie gar wohl als Kulturwert und Kulturmacht zu würdigen. Darum mahnt er selber den Katholizismus, "weun er sich nicht selbst zu einer quantité négligeable verurteilen will, an Kant, dem Schöpfer der kritischen Methode, dem Lehrer unbestechlicher Wahrheit und bisher unerhörter Denkschärfe, nicht gleichgiltig vorüberzugehen". So bezeichnet er, gleichsam programmatisch und allgemein, seine eigene Stellung zu Kant. Dem entsprechen auch Geberts besondere Darlegungen über die Kantische Philosophie.

Erfreulich berührt da zunächst die verständnisvolle Würdigung des Prinzips der Autonomie, rücksichtlich dessen sich der Autor ganz auf die Seite Kants stellt. Hatte der ultramontane Verstand, so wie eben diese Verstandesart Kant „verstehen“ musste, behauptet, dass dieses Prinzip der Autonomie „der Willkür Tür und Tor öffne", so zeigt Gebert, dass gerade im Gegenteil durch alle Heteronomie, ob sie sich nun unmittelbar als ${ }_{n}$ Glückseligkeitsstreben" äussere, oder ob sie das mittelbar in der Form von "Furcht und Hoffnung" tue - es liegt etwas vom Witz der Geschichte in dem eigentümlichen Zusammentreffen, dass Gebert die Worte des Ultramontanen längst vor diesem im entgegengesetzten Sinne gebraucht - „der Willkür Tür und Tor geöffnet

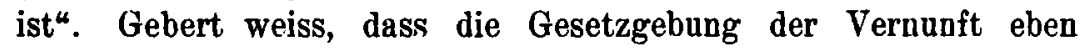
Gesetzgebung ist, dass das Gesetz der Vernunft objektiv bindend ist, dass „das Sittengesetz im Kantischen Sinne..., den Wünschen und Neigungen gegenüber mit der Anforderung der Pflicht auftritt“ und dass „der vernünftige Mensch ... sich diesem Gesetze

1) Vom Autor selbst gesperrt. 
freiwillig unterwirft". Zugleich erkennt er richtig, dass die allgemeine Vernunftgesetzlichkeit den Sinn ewiger Aufgegebenheit hat, dass die Gesetze der Vernunft ewige Aufgaben, die ewige Tätigkeit und Wirksamkeit fordern, bedeuten, und dass sie nichts zu tun haben mit dem, was sich die Gemächlichkeit des Ultramontanen als fertigen Besitz träumen mag. Vernunftgesetze dulden ja jene Gemächlichkeit nicht, von der Kant sagt, dass sie ,.schlimmer als alle Übel des Lebens“ ist.

Das Bewusstsein der unendlichen Aufgabe, das sich uns nicht bloss im Erkennen und in der Idee der Wahrbeit, die für den Ultramontanen, wie wir sahen, nur eine tote Sache sein kann, sondern vor allem auch in der Sittlichkeit und der Idee der Autonomie, erschliesst, von der der Ultramontane keine Ahnung haben kann, gilt Gebert, mit Recht nun auch „als Vorbedingung jeder wahren Religiosität". Diese ruht auch für Gebert auf der Autonomie, in der Kant ihre sittliche Grundlage entdeckt. Und so muss ihm, im echt christlichen Sinne, wie ihn Kant philosophisch begründet, die Religion nicht zu einem leeren autoritativen Bekennen, sondern zu einem innerlich überzeugungsvollen Erleben und einem lebendigen Betätigen nach der innerlich lebendigen Überzeugung werden. Die „religiöse Frfahrung“, das religiöse Erlebnis der sittlich-religiösen Persönlichkeit, wofür der Ultramontanismus nur seinen plumpen Spott übrig hat, der überzeugungsvolle persönliche Glaube, den der Ultramontane nicht besser zu behandeln weiss, als dass er ihn in Anführungsstriche setzt weil er sich nun einmal unter dem Glauben als lebendiger Überzeugung gar nichts denken kann und weil er sich selbst niemals überzeugen kann, sondern seinen ganzen Geistesinhalt von der Autorität empfängt - sie werden hier das Erste, und ausdrücklich wird erklärt, dass der blosse „Bekenntnisglaube an die zweite Stelle rückt".

So macht sich auch hier, ganz im Kantischen Sinne, eine reinere Fassung des Unterschiedes von Religion und Kirche, von der „Kirche Christi und ihrer zeitlichen Erscheinung“, wie Gebert sagt, bemerkbar, ein Unterschied, von dem der ultramontane Verstand natürlich wieder nichts verstehen kann. Ja selbst die Auffassung des Offenbarungsbegriffs und des Logmas erscheint durch die Kantische Lehre, die Gebert selbst als "einen Höhepunkt in der Religionsphilosophie" ansieht, geläutert und veredelt, so sehr, dass trotz des Katholizismus ein Zug zum Überkonfessionellen unver- 
Kant in neuer ultramontan- u. liberal-katholischer Beleuchtung.

kennbar ist, wenn er auch, wie wir noch sehen werden, notwendig nicht schon in reiner Gestalt hervortreten kann. Jedenfalls wird aber von den ultramontan-konfessionellen Quertreibereien treffend gesagt: „sie erwachsen nicht den treibenden Kräften des Geisteslebens, sondern einseitig hierarchischen Machtgelüsten und können nur dazu dienen, die Glaubenslosigkeit und, in den meisten Fällen damit zusammenhängend, die Religionslosigkeit zu vermehren."

Gegen die dogmatische Behandlung wird darum durchaus treffend ausgeführt: ,.So behandelte man und behandelt noch jetzt das Dogma als ein Primäres bei der religiösen Erziehung, als ob das Dogma zur religiösen Psyche, nicht vielmehr umgekehrt, die religiöse Psyche erst zum Dogma führte." Man sieht, wie Gebert das vom Ultramontanismus geschmähte religiöse Erlebnis von dessen dogmatischer Fixierung gar wohl unterscheidet. Ihn ist klar, wieweit der Weg vom unmittelbaren religiösen Bewusstsein bis zu dessen Fixierung im Dogma ist. wieviel auf diesem Wege verloren geht und wie armselig das Dogma gegenüber der unmittelbaren Religiosität und Sittlichkeit ist. Ihm liegt darum auch "der Schwerpunkt des Christentums nicht im Glauben als Bekenntnis, sondern in seiner lebendigen Betätigung, in dem Handeln aus Liebe zu Gott"; in dem Glauben also, der, nach dem Apostel Paulus, mit der Liebe eines ist, der also mit der Religion .eiue untrennbare Einheit in der Persönlichkeit ist". Man erkennt in dieser Glaubensauffassung deutlich die Abkunft von dem, allem Ultramontanismus so verhassten, praktischen Vernunftglauben Kants, wenn auch dieser selbst, wie wir bald zu bemerken haben werden, noch nicht erreicht ist. Freilich wird deutlich, dass diese Auffassung des Christentums nicht nur nicht die christliche Religion in den Gegensatz zur Kultur bringt, in den sie der Ultramontanismus setzt, es wird im Gegenteil die Bedeutung des Christentums für das Problem der Weltgeschichte, das durch jenes erst eigentlich entdeckt worden, erkannt, es wird weiter erkannt, dass das ecbte Christentum die Arbeit an allen Kulturwerten in sich beziehen kann, und dass sein rein innerlicher und verinnerlichter Überzeugungsglaube sogar - das ist ganz Kantisch - das Prinzip aller Kulturarbeit ist, und dass „ein Christentum, das sich der führenden Rolle im Kulturleben von selbst begiebt, das Vertrauen in seine geistige Durchschlagskraft verloren hat, mit dem wahren Christentum nur den Namen gemein" hat, dass nur eines Pseudo- 
christentums Maxime sein kann: „Abschliessung und geistige Inferiorität und kein Ende!“

Man sieht: für alle diese Ausführungen dürfte sich Gebert auf Kant berufen und dessen Einwirkungen auf die Anschauungen Geberts sind unverkennbar; und doch sind es die prinzipiellsten Punkte, rücksichtlich deren wir vom Standpunkte des Kantischen Kritizismus aus die vollendete Konsequenz vermissen. Zuuächst muss sich unsere Kritik gegen Geberts Auffassung - um an seine letzten Bemerkungen anzuknüpfen - vom Dogma richten. Es ist zwar schön und gut, das Dogma des Bekenntnisglaubens an die zweite Stelle gegenüber dem persönlichen religiösen Glauben als dem eigentlichen Glanbensprinzip oder Gesinnungsglauben zu rücken. ${ }^{1}$ ) Allein das Dogma selbst erscheint nach Geberts Ausführungen als solches doch immerhin noch unanfgebbar, überhistorisch und absolut. $\mathrm{Zu}$ einem adogmatischen Christentum hat sich der Reformkatholizismus Geberts also doch nicht hindurcbgearbeitet. Wir verspüren in seinen Anschaungen gewiss den Hauch eines überkonfessionellen Triebes, und über den Konfessionalismus ultramontaner Observanz ist er hoch erhaben. Allein ein vollkommen und bewusst überkonfessionelles Christentum wäre doch erst mit der prinzipiellen Preisgabe des Dogmas, das ja als solches gerade die Konfession bestimmt, erreicht. Aber gerade das adogmatische Christentum ist in letzter Linie doch das der philosophischen Begründung allein fähige, und darum auch das Christentum im Sinne Kants. Und so liegt denn, trotz aller Verwandtschaft und Abhängigkeit auf der einen Seite, dennoch auf der anderen Seite zwischen der reformkatholischen und der kritisch-philosophischen Anschauung eine ganze Welt, eben die Welt des Dogmas.

Sodann aber besteht ein ebensowenig überbrückbarer, darum auch von Gebert nicht ausgeglichener Gegensatz zwischen dem von Gebert freilich mit grossem Nachdruck geforderten Prinzip der Autononie und dem anderen Prinzip, dem sich wohl auch der Reformkatholik nicht entziehen kann, solange ihm noch daran liegt, von den kirchlichen Mächten in irgend einer Weise wenigstens anerkannt $\mathrm{zu}$ werden, nätnlich dem Prinzip der Autorität. Duldet die Autonomie in letzter Linie keine dogmatische Bekenntnisreligion, so duldet sie auch keine das Dogma hütende Religions-

1) Uber diese Unterscheidung vgl. genauer meine Schrift: Luther und Kant, S. 20 ff. 
autorität. Freilich wendet sich Gebert gegen die blosse „Heeresfolge" der Kirche, auch gegen die „äussere Autorität". Solange es aber möglich ist, dass - nicht etwa bloss ein Glossner einem Gebert als kirchliche Autorität zu gelten hat, nein vielmehr dem System uach der Reformkatholizismus überhaupt eine Autorität in Glaubenssachen anerkennt, solange er nicht, und das kann er wohl nicht, um auch nur Reformkatholizismus zu bleiben, aller Autorität klipp und klar seine Absage giebt, solange ist die von ihm selbst geforderte Autonomie unrealisierbar. Autonomie und Autoritätsglaube, welcher Art dieser auch sei, schliessen sich in alle Wege aus. Fs ist darım nach den Prinzipien kritischer Religionsphilosophie nicht genug, das Dogma gegenüber der Religion an die zweite Stelle zn rücken und ihm doch einen starren Gehalt zuschreiben zu wollen; ebensowenig ist es genug, der Autorität $z w a r$ die Alleinherrschaft $z u$ nehmen und ihr dennoch ein, wenn auch beschränktes, als solches aber bleibendes Herrschaftsrecht einzuräumen. Die Prinzipien der kritischen Philosophie fordern, das Dogma lediglich als den zeitlichen Ausdruck eines religiösen Gehaltes anzusehen, ein Ausdruck, eine Form, die wegen der zeitlichen Bedingtheit aber niemals für alle Zeit bindende Kraft haben kann, sondern, um Kantisch zu reden, bloss als ein "Vehikel" zu betrachten ist, das mau, wie allen Schriftglauben, muss „dereinst entbehren können“, um dem Prinzip des ,guten Lebenswandels" als dem alleinigen sittlich-religiösen Prinzip vollkommen freien Raum zu geben. Und ebenso fordern die Prinzipien der kritischen Philosophie, um die Idee der Autonomie zur Entfaltung gelangen zu lassen, dass das Gängelband der Autorität auf sittlich-religiösem Gebiete nicht bloss etwas gelockert, sondern, dass es endlich vollkommen und radikal abgeworfen werde. Das sind die Forderungen Kants, die nie aufgegeben werden dürfen, soll der Weg der Geschichte wirklich ein Weg approximativer Annäherung an das Ideal, die geschichtliche Arbeit wenigstens eine Form der Darstellung des Ewigen in der Zeit sein.

So interessant die Gebertschen reformkatholiscben A usführungen, so achtunggebietend die edlen Bestrebungen des Autors, so herzerfreuend ihre Gegensätze zuın Ultramontauismus auch sein mögen, so hat das alles doch mein Urteil über den Reformkatholizismus, das ich früher schon bei anderer (jelegenbeit ${ }^{1}$ ) ausgesprochen und

1) Luther u. Kant, S. 190. 
das sich auch mit dem Pfleiderers ${ }^{1}$ ) deckt, nicht modifizieren, sondern nur bestätigen können. Wie Pfleiderer, so erkenne ich, wie schon gesagt, gerne den edlen Sinn dieser Bestrebungen an, nicht minder die lautere Absicht, im allgemeinen Kulturzusammenhange mitzuarbeiten und mitzuwirken. Allein sie bleiben dennoch auf halbem Wege stehen. Freilich ist heute noch keine sichtbare religiöse Gemeinschaft - das mag vielleicht ein Trost für den Reformkatholizismus sein, der aber vor dem Forum der kritischen Philosophie nicht besteben kann, weil diese nicht Trostgründe, sondern Wertgründe zn suchen hat - soweit, das Autonomieprinzip und das adogmatische Christentum rein ausgeprägt zu haben. Auch das protestantische Kirchentum hat, wie das Pfleiderer sehr treffend betont, solange das protestantische Prinzip noch nicht rein dargestellt, als es sich von der Bekenntnisformel nicht frei gemacht, von dieser noch die Zugehörigkeit abhängig macht und so gar manchen protestantisch Denkenden von sich ausschliesst. In dieser Hinsicht gilt heute noch, was zu Kants Zeiten galt, und was Kant mit unzweideutiger Klarheit kritisch bestimmt. Allein der grosse Unterschied ist doch der, dass im protestantischen Prinzip die Idee der unsichtbaren religiösen Gemeiuschaft selbst eben zum Prinzip erhoben ist. Mag heute noch das konkrete Leben der Wirklichkeit darum vom Prinzip noch so weit entfernt sein, so ist in dem Verhältnis von Ideal und Leben doch zuerst da etwas für die gestaltende und richtunggebende Macht der Idee auf das Leben zu hoffen, wo die Idee wenigstens im Bewusstsein ergriffen ist, auch wenn die konkrete Lebensgestaltung sich ihr noch nicht gefügt hat. Aber damit diese sich ihr füge, dazu ist die unerlässliche Bedingung, dass sie sich als Prinzip in der "reinen Erkenntnis" erst befestige.

Das ist das Urteil, zu dem die sachliche Kritik der theoretischen Ausführungen Geberts gelangen muss. Mag er theoretisch fürs erste auch noch auf halbem Wege stehen geblieben sein, die persönliche redliche und ernste Bemühung um die innere sittlichreligiöse Freiheit ist nicht zu verkennen. Und wenn seine theoretischen Ausführungen auch noch nicht die letzten logischen Konsequenzen gezogen, so scheint mir das bei dem redlichen persönlichen Willen doch nicht daran gelegen zu haben, dass ihm der Mut zu den

1) In seiner Abhandlung über den Reformkatholizismus (in der Monatsschrift „Deutschland", Aprilheft 1903). 
Konsequenzen gefehlt, sondern daran, dass in dieser Schrift eben nur ein erster Versuch vorliegt, der noch nicht zu vollkommen theoretischer Eutfaltung gelangen konnte. Mag darum Geberts Theorie rom Standpunkte der kritischen Philosophie noch gar manches vermissen lassen, mag sie, wie wir sagten, auf halbem Wege stehen geblieben sein, so lässt doch praktisch seine lantere persönlicheGesinnung gerade von ebendem Standpunkte der kritischen Philosophie, der die Gesinnung wohl zn würdigen weiss, doch der Erwartung Raum, dass sein theoretiscber Standpunkt noch kein definitiver ist, dass er seinen Weg weitergehen und zu einem Ziele gelangen, dass er in der kritischen Philosophie nicht bloss einen Bundesgenossen gegen den verderblichsten Feind aller Geisteskultur, gegen den Ultramontanismus, sondern die Methode finden wird zur Erarbeitung eines durchaus eigenen geistigen Lebensinhaltes nach dem Prinzip der sittlichen Freiheit.

\section{Nachtrag.}

Die vorstehende Abhandlung war bereits im Druck vollendet, als ich auf einige Publikationen aufmerksam wurde, die den gleichen Gedankenkreisen angehören und darum klar und deutlich zeigen, dass wir es in den besprochenen Erscheinungen nicht mit etwas Vereinzeltem, sondern mit etwas Typischem zu tun haben. Dass dieses auch als solches wieder nicht auf unser deutsches Vaterland beschränkt bleibt, beweist eine gleich in zwei Zeitschriften, in der Revue Thomiste und in der Revue de Philosophie, geführte Kant-Kontroverse zwischen zwei katholischen Theologen, einem Abbé Farges und dem den Lesern der Kant-Studien ja nicht unbekannten C. Sentroul. Herr Farges deutet in echt ultramontaner Art wieder Kants Lehre zum "Subjektivismus" aus und liefert eine höchst unglückliche „Refutation“. Seine Art, Kant zu "refutieren" bewegt sich, dem wissenschaftlichen Ethos nach, zwar in etwas vornehmeren Bahnen, als diejenige Herrn Glossners. Ein gleiches Niveau wäre ja auch dem radikalsten Ultramontanen schwer, ein tieferes aber unmöglich. Das Ziel dieser Bahnen ist indes dasselbe, wie das Herrn Glossners. Wie dieser, so ist auch Herr Farges ultramontan. Nur die Art des Gedankenausdrucks ist durch die französische Förmlichkeit um einige Grade gemässigt. Herr Sentroul wiederum ist in seiner Liberalităt ganz erheblich zurückhaltender, als Gebert, der schon als antiultramontaner Deutscher dem Begründer des Autonomieprinzips durch theoretische und praktische Sympathien inniger verbunden sein musste, als der belgische Neu-Scholastiker, der im Herzen ja ein guter Aristoteliker geblieben ist. Immerhin knüpft sich ja an Sentrouls Namen die Tatsache, dass er uns unter den erfolgreichen Bewerbern um den Kant-Preis begegnet ist, ein Ereignis, das immerhin bemerkenswert ist, wenn man bedenkt, dass Herr Sentroul katholischer Theolog und Priester ist. Freilich spricht das noch nicht, wie aus 
der Kritik der Preisrichter selber hervorgeht, für ein besonders inniges Verhältnis zu Kant, doch aber dafür, dass Herr Sentroul sich wenigstens um ein Verständnis für die Lehre des grössten Denkers der Neuzeit trotz seiner persönlichen aristotelisch-scholastischen Neigungen und Überzeugungen bemüht hat. Belustigend aber wirkt es, dass Herr Sentroul seine erfolgreiche Preisbewerbung vom strikten Thomisten sich nun auch noch vorrücken lassen muss. Er wird an seinem Preise wohl überhaupt nicht leicht zu tragen haben. In der Meinung der strengen Orthodoxie seiner Kirche drückt dieser Preis dem neu-scholastischen Priester wohl gar einen Makel auf, und wer mit der kritischen Philosophie eine etwas innigere Fühlung hat, als der Neu-Scholastiker, der wird vielleicht bedauern, dass nur einem Herrn Farges Sentrouls Verhältnis zu Kant als ein zu inniges erscheint, wahrend in Wahrheit doch der Geist der kritischen Philosophie in Sentrouls Denkweise kaum besonders Macht und Nachdruck gewonnen hat und hinter dem Scholastizismus weit zurücksteht. Herrn Farges gegenüber bemüht sich Sentroul freilich in einer an und für sich ganz erfreulichen Weise um eine Aufklärung über den vermeintlichen -Subjektivismus" Kants; aber doch mit um so grösserer Vorsicht und Reserve, als er selbst über die subjektivistische Deutung nicht erheblich hinausgelangi ist. Und dennoch fürchten wir, dass alle Mühe um eine selbst sehr zurückhaltende Aufklärung dem ultramontanen Abbé gegenüber vergeblich aufgewandt sei. Leider ist auch im Ganzen trotz mancher Differenz im Einzelnen der Gegensatz zwischen Sentroul und Farges viel weniger scharf, als der zwischen Gebert und Glossner. Innerhalb der katholischen Gläubigkeit finden die Extreme der Stellungnahme zu Kant, wie sie bei Gebert und Glossner vorliegen, in dım Streite von Sentroul und Farges eine gewisse, nicht gerade erfreuliche Berührung, sodass die Bedeutung dieser ausländischen Kontroverse eigentlich $\mathrm{zu}$ einem blossen katholisch-intratheologischen Schulstreite herabsinkt.

Von mehr philosophischem, wenn auch leider ebenfalls mehr bedauerlichem Interesse ist wieder eine Invektive gegen Kant, die von einem Ultramontanen deutscher Zunge ausgeht, und die in dem dritten Bande der zweiten Auflage von Willmanns Geschichte des Idealismus vorliegt.1) Fritz Medicus hat in den Kant-Studien ${ }^{2}$ ) in der Abhandlung "Zwei Thomisten contra Kant", so eingehend und treffend uber die erste Auflage dieses Buches berichtet, dass uns über die, wenigstens der allgemeinen Tendenz nach, nicht erheblich veränderte zweite Auflage nicht viel zu sagen bleibt. Was das Buch in der ersten Auflage war, ist es im ganzen anch in der zweiten geblieben: "ein“, wie Medicus sagte, „bedrohliches Zeichen der depravierenden Matht des Ultramontanismus". Die zweite Auflage bezeichnet sich zwar als "verbesserte". Aber die „Verbesserung“ steht bloss auf dem Titel. Auf den Inhalt des Buches hat sie sich so wenig erstreckt, dass nicht einmal die der ersten Auflage bereits von Medicus nachgewiesenen Quartanerschnitzer auf dem Gebiete der Elementargeome-

1) Geschichte des Idealismus von Dr. Otto Willmann, K. K. Hofrat, Universitatsprofessor i. R. 2. Aufl. III. Bd. Braunschweig. Druck und Verlag von Friedrich Vieweg \& Sohn. 1907.

7) Kant-Studien III, S. $326 \mathrm{ff}$. 
trie - alles Mathematische scheint für Willmann überhaupt eine ebenso grosse crux zu sein wie alles Philosophische - ausgemerzt sind, mit denen gegen Kant so lustig operiert wird. Es ist in der Tendenz des ganzen Buches so sehr alles beim Alten geblieben, dass wir es hier nicht mehr mit einer neuen ultramontanen Beleuchtung der Kantischen Lehre zu tun haben, sondern nur mit einer neuen Projektion des alten ultramontanen Bildes, das Willmann bereits vor Jahren entworfen hatte. Aber ganz achtlos dürfen wir vielleicht doch auch diesmal nicht an der immerhin charakteristischen Erscheinung vorbeigehen.

Herr Willmann redet über Kant ja nicht, wie Herr Glossner, d. h. wie der Blinde über die Farben. Er hat ihn. wenigstens gelesen. Aber er spricht über Kant auch nicht, wie der Physiker und der Physiologe über die Farben, sondern wie das Kind, das von der physikalischen und physiologischen Optik keine Ahnung hat, das also von den Farben, von denen es spricht, nichts versteht. So versteht Willmann auch nichts von Kant. Die Kindlichkeit beschränkt sich freilich nur auf dieses Nicht-Verstehen. Denn die Mittelchen, mit denen die ultramontanen Leser - dass sie auf urteilsfähige Leser wirken könnten, wird Herr Willmann selber nicht glauben - gegen Kant bearbeitet werden sollen, wird man wenigstens praktisch nicht gerade als kindlich bezeichnen können, wenn sie auch darauf abzielen, den Ultramontanen das Gruseln vor Kant zu lehren: "Hochmut", "Verstiegenheit", „Verblendung“, „Verschrobenheit", „Pietätlosigkeit", „Hoffart", „Unbotmässigkeit", „Überhebung“, „Selbstherrlichkeit", "der orgiastische Aufruf zur Selbstanbetung", „Zynismus", ,Aftermoral“, - das alles sind Prädikate, die Kant besudeln sollen. Weil aber damit der ultramontanen Absicht wohl immer noch nicht genug gedient ist, und damit die gläubige Lesergemeinde Willmanns nun auch wirklich und ausgerechnet drei Kreuze vor dem Ungeheuer schlage, so muss Kant, dem wohl gegen ein Dutzend Mal "Sophistik" vorgeworfen wird, erstens als „völlig irreligiős", zweitens als "Atheist", drittens als "moralisierender Anarchist" gebrandmarkt werden. Kann man von einem solchen ${ }_{n}$ völlig irreligiösen" ${ }_{n}^{\text {Atheisten }}$ und „moralisierenden Anarchisten ${ }^{\star}$ auch nur ein Minimum von „Ehrlichkeit" erwarten? Ja, mit Kants „Ehrlichkeit" treibt Herr Willmann ein seltsames Spiel. An die bekannte Kantische Erörterung über das Gebet hatte Willmann in der ersten Auflage die Bemerkung geknüpft: „Die ganze Hoffart, Verlogenheit und Heuchelei der Aufklärer spricht aus diesen Worten, die zugleich ein grelles Schlaglicht auf die Ursachen der sozialen Dekomposition des protestantischen Deutschlands werfen". Trotzdem aber will Herr Willmann Kants Ehrlichkeit "nicht "in Frage" gezogen haben. Das sei ein „Missverständnis“ der „Kritiker" gewesen. Nichtsdestoweniger heisst es aber doch in der zweiten Auflage wieder im Anschluss an jene Kantische Stelle über das Gebet: „Den Aufklärern hat man oft Hoffart, Verlogenheit und Heuchelei schuldgegeben. Ist das angesichts solcher Äusserungen zu verwundern? Äusserungen, die zugleich ein grelles Schlaglicht auf die Ursachen der sozialen Dekomposition des protestantischen Deutschlands werfen." Ja, ja, die Kritiker haben Herrn Willmann schon so verstanden, wie ihn freilich nur die Oltramontanen verstehen sollten. Nur reicht das Gedăchtnis der Kritiker etwas weiter, 
als das der ultramontanen Leser, die vielleicht vergessen haben mögen, was ihr Orakel 90 Seiten vorher verkündet; was der Kritiker aber festnagelt. Und wenn Herr Willmann Kant gegenüber von "geistiger Falschmünzerei - wenngleich ohne dolus" - redet, nun so wird der Kritiker freilich zugeben, dass der Begriff der Ehrlichkeit, wie ihn Herr Willmann fasst, allerdings leicht zu einem "Missverständnis" führen kann, ja dass er diese Auffassung von Ehrlichkeit wohl überhaupt nicht recht zu verstehen im Stande ist. Blosse „Veränderungen des Ausdruckes" und eine blosse "Erklärung“ genügen nicht, damit einem solchen „Missverständnis“ „abgeholfen" werde, sofern der Kritiker selbst auf dem Standpunkte der autonomen Ethik steht; und es liegt wohl in letzter Linie an diesem Standpunkte, dass er jene Auffassung von Ehrlichkeit "missverstehen" muss.") Soll er aber Kant gegen solche Vorwürfe in Schutz nehmen? Nein, gewiss nicht. In diesem Punkte hat bereits Paulsen in seiner vornehmen und gerechten Kritik der ersten Auflage dieses Buches so sehr das Rechte getroffen und in aller Kürze treffend zum Ausdruck gebracht, dass wir auf die Anschuldigungen, die Willmann gegen Kant erhebt, Paulsens Antwort ohne Einschränkung zu der unsrigen machen können: „Ich meine, nicht die Ehre Kants ist es, die hierbei leidet, sondern die Ehre dessen, der sich so an ihm vergeht."2)

Genau so verzerrt und entstellt, wie das Bild der Persönlichkeit unseres grössten Denkers, ist auch seine Lehre. Soviel Sätze der Autor in diesem Buche über die Kantische Philosophie niedergeschrieben hat, soviel Unsinn ist auch darin zusammengetragen. Und die personnlichen Verdächtigungen, die dem ahnungslosen Leser stetig versetzt werden, mögen recht geeignet sein, dabei mitzuhelfen, dass auch das Bild der Lehre in dem Lichte erscheine, in dem es eben nach dem Wunsche ultramontaner Zurechtmachung gesehen werden soll.

Wie freundlich Herr Willmann zu ihr steht, und welches Schicksal er ihr wünscht, das hat er uns an einer Stelle seines Buches unbedacht verraten. Er meint einmal, wenn Kant sich zu der scholastischen Auffassung vom Begriff des Noumenons bekehrt hätte, dann würde er „die Vernunftkritik ins Feuer geworfen" haben. Nun diese scholastische Auffassung ist, wie jeder weiss, der Kant verstanden hat, Unsinn. Aber sie ist auch Herrn Willmanns Auffassung. Kants Bekehrung indes ist unterblieben. Der Ketzerrichterwunsch, "die Vernunftkritik ins Feuer geworfen" zu sehen, illustriert aber immerhin einigermassen die ,geistigen" Waffen des Ultramontanismus; und aus der Geschichte ist ihm ja deren erfolgreicher Gebrauch wohl bekannt, aus Zeiten, wo das Feuer ein machtvolles

1) Logisch kann man darum aber diesem Begriffe sehr wohl beikommen. Das zeigt am besten die Art, auf die Schuppe in den Anwendungen seiner grossen erkenntnistheoretischen Logik das tut. Es ist an und für sich schon interessant, zu bemerken, für welche logischen Fragen die ultramontane Logik das Anwendungsmaterial hier geliefert hat. Gradezu glänzend aber ist die Prüfung, die hier die Prinzipien der ultramontanen Logik an den Kriterien wirklicher Logik finden.

9) Philosophia militans S.17 (vgl. dort die Abhandlung: „Das jüngste Ketzergericht tber die moderne Philosophio*). 
Kant in neuer ultramontan- u. liberal-katholischer Beleuchtung.

,Argument" in Sachen wissenschaftlicher, wie religiöser Überzeugung war. Ich erwähne das nicht willkürlich, sondern nur um die Geistesart dieses Kampfes auch gegen die Lehre, nicht bloss gegen die Persönlichkeit Kants zu beleuchten. Nur von der mittelalterlichen Anschauungsweise her ist dieser Kampf gegen die moderne Philosophie, gegen die philosophische Seite des „Modernismus" zu verstehen. Mit mittelalterlichen Mitteln wird auf der einen Seite die Kantische Lehre verzerrt, und mit mittelalterlichen Mitteln wird sie auf der anderen Seite bekämplt. Für die ganze mittelalterliche Verzerrung des Geschichtlichen ist schon die ganze Tendenz charakteristisch, mit der der Kritizismus auf einen begrifflichen Ausdruck gebracht werden soll. „Die Subjektivierung des Idealen durch Kants Autonomismus" - das ist der Titel, der Kants Lehre aufgeheftet wird. Die Autonomie wird als der Nerv der Kantischen Lehre erkannt. Weil aber die Autonomie in ihrem Wesen völlig verkannt wird, darum muss mit Notwendigkeit auch die ganze Kantische Lehre als „Subjektivierung des Idealen" verkannt werden; in ihrem praktischen, wie in ihrem theoretischen Teile.

Der durch die persönliche Verunglimpfung zu Tage tretenden fanatischen Absicht entsprechend, scheint Willmann in seiner aller logischen Anordnung entbehrenden Darstellung den persönlichen Nachdruck auf den praktischen Teil zu legen. Da er nur klerikal-scholastisch zu denken im Stande ist, von der wahren Objektivität des Idealen also keine Ahnung haben kann, so muss ihm natürlich wieder auf echt ultramontane Art die Autonomie zur Willkür werden. Sie wird ihm gleichbedeutend mit „,schrankenloser Willkür", mit der Tendenz des „Übermenschen“. Was Wunder, wenn es bei ihm heisst: „Der Autonomismus ist seiner Natur nach Egoismus". Also nicht einmal die ersten Sätze der Kritik der praktischen Vernunft, die zum Einfachsten gehören, was Kant je geschrieben hat, hat Herr Willmann verstanden. Für die kritische Objektivităt fehlt ihm jeder Sinn, weil klerikale, autoritative, scholastische Schein-Objektivität sein Denken gefangen genommen hat. Hören wir ihn selber: „Von einem Halt kann ja der Autonomismus nichts wissen - denn woran ich mich halte, das muss ausser mir sein, also meinen Willen heteronomisch bestimmen.“ Mit diesem sensualistischen, der Sprache entnommenen „Arguinent" glaubt der kirchenmoralistische Thomist wirklich eine Objektivität erhärten zu können; die ideal-kritische ist das wahrlich nicht, und darum ist es überhaupt keine. Es ist ein kläglicher Schein von Objektivităt, den als solchen nur der klerikale Dogmatist nicht zu durchschauen vermag.

Wie horrend nun auf theoretischem Gebiete das Verkennen der kritischen Objektivitat ist, das beweist am besten vielleicht der Umstand, dass Herr Willmann gerade im Anschluss an eine Stelle, in der Kant mit einer jedem Denkfähigen einleuchtenden Klarheit und Einfachheit allen Subjektivismus und Illusionismus abweist, seinen gläubigen Lesern nun das Märchen auch vom theoretischen Subjektivismus auftischt. Er kann aber das Ideale immer nur verkörperlicht fassen, wie seine klerikal-scholastischen Vorgänger, die den tiefen Sinn der platonischen Lehre durch die Verkörperlichungssucht zu ertöten versucht hatten. Ein Versuch, der wohl auch mit der Zeit noch gelungen wäre, wenn die Geschichte nicht 
gerade un Kant gelernt hatte, Platon die, durch die mittelalterliche Kirche solange vereitelte und durch ihren nachwirkenden Einfluss noch in die Neuzeit hinein hintangehaltene, Gerechtigkeit widerfahren zu lassen. Der Aristoteliker unserer Tage kann Platon noch weniger verstehen, als der Aristoteliker des Mittelalters. Am wenigsten aber kann er Kant verstehen. Ihm müssen natürlich Kants Kategorien zum blossen „Plunder“ werden, und sie, wie die Anschauungsformen, zu einer Art subjektiver Funktion, durch die wir den Dingen „unseren Stempel aufprägen“.

Diesen thomistischen Deutungsmitteln entsprechen auch die Widerlegungsmittel. Soweit überhaupt von Argumenten die Rede ist, und sich die "Widerlegnng" nicht mit den erwähnten persönlichen Prädikaten begnügt, sind jene abgestandene scholastische Mittelchen Die stillschweigende Voraussetzung ist immer - oft auch die ausgesprochene -: Der heilige Thomas hat Recht. Damit ist von vornherein die . Wahrheit als fertiger Besitz der Kirchenlehre verbürgt. Nun befindet sich Kant im Widerspruch mit der Kirchenlehre und dem heiligen Thomas. Das zu zeigen ist leicht, und ebenso leicht ist der Schluss: Also hat Kant Unrecht. Das ergiebt sich ebenso leicht, wie der Nachweis von Kants "Hochmut", wenn man einmal erst voraussetzt, dass autonomes Denken Hochmut ist. Denn Kant ist autonom im Denken. Darum ist er hochmütig. Also schliesst Herr Willmann. Wir würden freilich von anderen Prämissen ausgehen und etwa sagen: Wer auf autonomes Denken verzichtet, der liat in der Wissenschaft überhaupt nicht mitzureden. Das Subjekt des Untersatzes mag vertreten, wer es auch sei, und wäre es auch traurigerweise ein Professor der Philosophie. Also - so würden wir schliessen, Herr Willmann.

Herr Hofrat Willmann spricht einmal mit Entrüstung von „Büblein“", die "mit Steinen" nach den ewigen Ideen geworfen, natïrlich unbeschadet der Bedeutung und Geltung der Ideen. Dass er dabei wieder auf Kant abzielt, versteht sich. Aber er weiss nicht, was er tut. Darin freilich hat er vollkommen Recht, dass die ewigen Ideen durch Bubenstücke keinen Schaden leiden. Und so Recht hier Herr Willmann hat, ebenso wahr wird auch der Erkenntnis- und Sittlichkeitsgehalt des Kantischen Werkes bleiben und seine Wirkung tun, ob auch der Ultramontanismus sich mit tausend und abertausend Bubenstücken an ihm versündige. Alle ultramontane Verdunkelung kann durch Kontrastwirkung das Licht der Vernunftkritik nur um so heller erstrahlen und darum auch mit zur Wirkung gelangen lassen, so wahr in der Geschichte der Menschheit ein ewiger Sinn lebendig und wirksam ist. So wird sich die Autonomie des Denkens und Wollens - mag sie vielleicht auch wie der Protestantismus selbst, aus dem sie hervorgewachsen ist, in Rom als "Pest" bezeichnet werden - nie wieder aus dem Bewusstsein der Menschheit verdrängen lassen, und ,wenn die Welt gleich voller Päpste wär‘. 